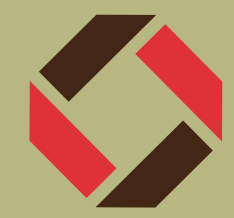

\title{
Discovery and rediscovery of gutta percha, a natural thermoplastic
}

\section{By Abbas Bacha and Hortense Le Ferrand}

$\mathrm{T}$ he age of fast, modern, and globalized networks of communication started in the late 19th century with the development of an electrical network and telegraphy. In 1858, cross-continental communications between North America and Europe were completed in minutes thanks to cables running on the bottom of the ocean. At the origin of this technological achievement is a humble tree sap called gutta percha, which presents properties not found in synthetic materials at the time. Almost forgotten today, gutta percha may bring new promise as a natural and sustainable material.

Gutta percha is a latex gum extracted from the sap of a Palaquium family of trees (see Figure a, b). Until its use for transatlantic cables, this resin had been known and used by local communities of the Malayan Peninsula to craft handles for machetes and other utility tools. Its existence had been limited to the region up until 1843, when a surgeon from the East India Company, William Montgomerie, wrote about this unique material and suggested its use in medical instruments. Gutta percha presents interesting properties: it is a natural rubber that is soft, moldable in hot water, and that becomes hard when cooled, like a thermoplastic. However, one particularity of gutta percha is its chemical structure, based on the polymerization of a stereoisomer of isoprene, the monomer forming natural rubber (see Figure c). This chemistry leads to higher crystallinity, around 30-40\% hydrophobicity, with a contact angle with water of ca. $94^{\circ}$ and a higher melting temperature of ca. $50-70^{\circ} \mathrm{C}$, as compared to ca. $30^{\circ} \mathrm{C}$ for isoprene while maintaining a similar glass transition temperature around $-70^{\circ} \mathrm{C}$. As a result, gutta percha is less extensible but stiffer than natural rubber, with an elongation at break of around $300 \%$, as compared to $650 \%$ for rubber, but has a Young's modulus around $100 \mathrm{MPa}$ as compared to $2 \mathrm{MPa}$ for rubber.

Gutta percha became truly important as an industrial material when it was heavily used as an easily processable material for electrical and water insulation for submerged telegraph cables. It was also used for the first phonograph records, before being replaced by vinyl, and is still used today in niche applications, such as dental fillings or golf ball cores. Because of its popularity for electric cable protection, more than $370,000 \mathrm{~km}$ were laid out

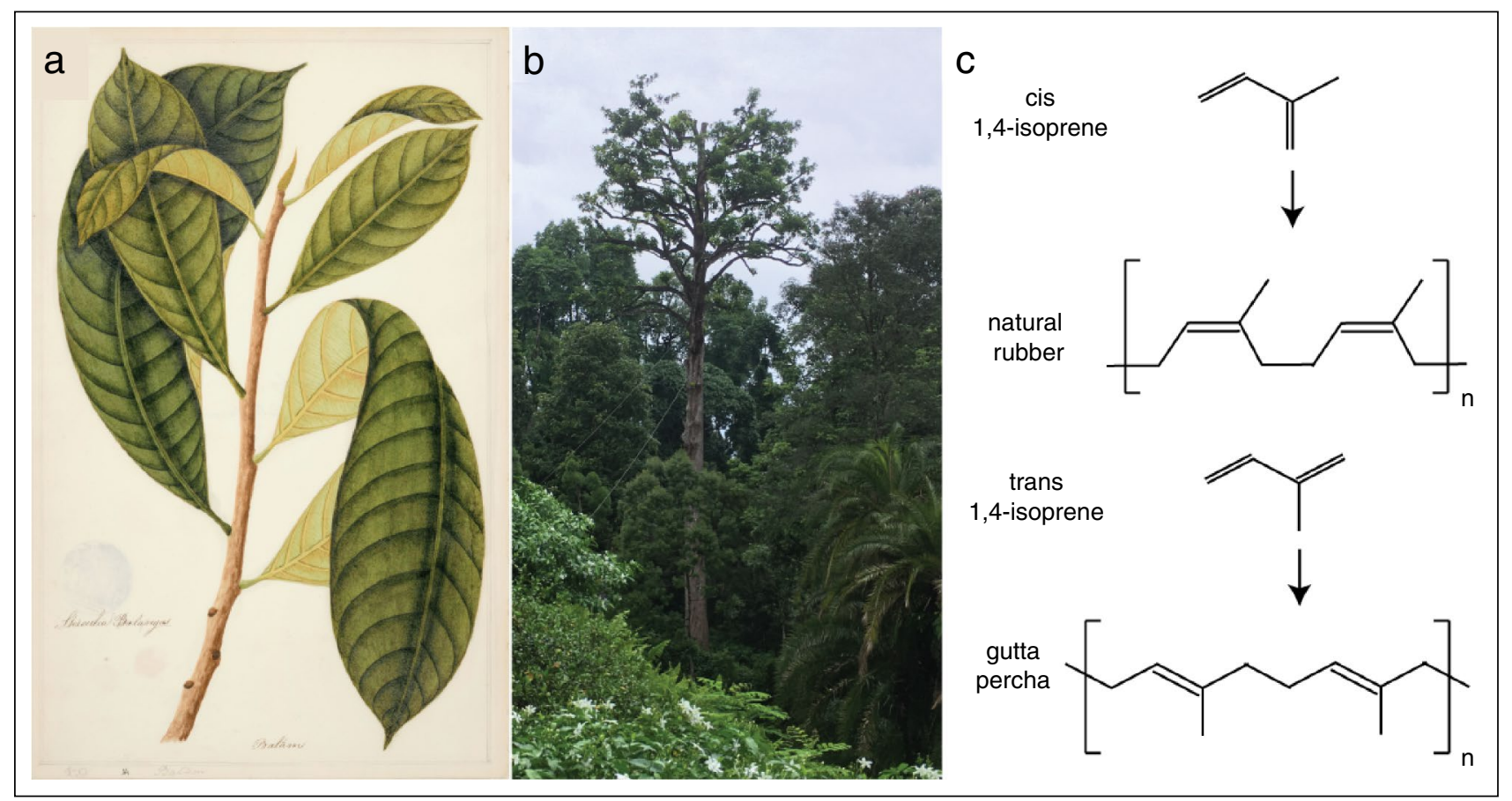

Figure. (a) Watercolor of the leaves of Nyatuh Putih, the indigenous name of the gutta percha tree, from the Collection of Natural History Drawings of William Farquhar, Scottish employee at the East India Company, 1803-1818. (Courtesy of the National Museum of Singapore, National Heritage Board. Gift of G.K. Goh). (b) Picture of a century-old gutta percha tree. (c) Chemical structures of natural rubber and gutta percha latex. 
by 1900 . Although this was an enthusiastic and impactful development, the industry endangered the species because of unsustainable extraction methods, which killed the trees during the process. With the naïve thinking that trees are an infinite resource, they were ruthlessly exploited until close to the extinction of the species. This ecological disaster was then forgotten by the introduction and development of thermoplastics and their massive production and use.

Yet gutta percha still holds promise, particularly as a sustainable material with water resistance and durability. Current research proposes the use of composites made of gutta percha and additives for agricultural protective films, purifying membranes, or for photothermal conversion. For example, composites of biological charcoal and gutta percha showed an increase in stretchability and low water vapor permeability while maintaining high tensile strength. When compared to its synthetic competitor, polyethylene, the biocomposites exhibit a higher biodegradability that makes them suitable as protective films around crops in agriculture. Similarly, the addition of carbon nanotubes increases the stretchability of gutta percha films up to nearly $400 \%$, while slightly increasing the Young's modulus and maintaining the tensile strength, around $100 \mathrm{MPa}$ and 15-20 MPa, respectively. In those composites, photothermal conversion increases, enabling soil warming. Furthermore, gutta percha's hydrophobic properties, due to its aliphatic chemical nature, can also be capitalized for the development of oil and solvent capturing membranes. Incorporating gutta percha into silylated nanofiber membranes shows increased efficiency in the

\section{Further reading}

1. J.S. Gamble, Gutta percha trees of the Malay peninsula. Bull. Misc. Info. 4, 109 (1907).

2. J. Tully, A Victorian ecological disaster: imperialism, the telegraph, and guttapercha. J. World Hist. 20, 559 (2009).

3. D. She, J. Dong, J. Zhang, L. Liu, Q. Sun, Z. Geng, P. Peng, Development of black and biodegradable biochar-gutta percha composite films with high stretchability and barrier properties. Compos. Sci. Technol. 175, 1 (2019).

4. Y. Yang, P. Peng, Q. Yang, D. Wang, J. Dong, Fabrication of renewable gutta percha/silylated nanofibers membrane for highly effective oil-water emulsion separation. Appl. Surf. Sci. 530, 147163 (2020).

5. H. Yang, P. Peng, Q. Sun, Q. Zhang, N. Ren, F. Ha, D. She, Developed carbon nanotubes/gutta percha nanocomposite films with high stretchability and photothermal conversion efficiency. J. Mater. Res. Technol. 9, 8884 (2020).

6. X. Men, F. Wang, G.Q. Chen, H.B. Zhang, M. Xian, Biosynthesis of natural rubber: Current state and perspectives. Int. J. Mol. Sci. 20(1), 50 (2018).

7. R. Chen, Y. Harada, T. Bamba, Y. Nakazawa, K. Gyokusen, Overexpression of an isopentenyl diphosphate isomerase gene to enhance trans-polyisoprene production in Eucommia ulmoides Oliver. BMC Biotechnol. 12, 78 (2012).

separation of the hydrophobic components such as petroleum ether, dichloromethane, cyclohexane, and toluene from oil-in-water emulsions. Finally, the thermoplastic properties of gutta percha also make it an appealing base bioresin for 3D printing. Since its biocompatibility in endodontic applications is already known, it may be a good candidate for new and innovative $3 \mathrm{D}$ printable resins for biomedical applications.

If the rediscovery of gutta percha opens innovations for new sustainable materials, viable ways of its cultivation and extraction that exclude the use of fossil fuels should be practiced. Currently, biosynthesis of isoprene from biomass fermentation is of interest in the synthetic biology community. Yeast and bacterial cells have already been successfully engineered to produce isoprene from waste biomass, especially cellulose. Genencor, a biotech company in the United States, has successfully converted this process and is producing isoprene from fermentation at a commercial scale. Alternatively, latex-producing plants may be engineered to increase their output by identifying the metabolic pathway of trans-polyisoprene production in these plants. This has been demonstrated with Eucommia ulmoides, a plant that also produces trans-polyisoprene. The plant was modified to overexpress isopentenyl diphosphate isomerase, ultimately leading to a three to fourfold increase in overall trans-polyisoprene content. Other efforts are under way to engineer transgenic yeast that produces rubber in vitro. These processes are scalable and could allow gutta percha to be a viable and promising alternative to latex production based off petroleum feedstock for a large diversity of applications.

We welcome comments and feedback on this article via email to Bulletin@mrs.org.

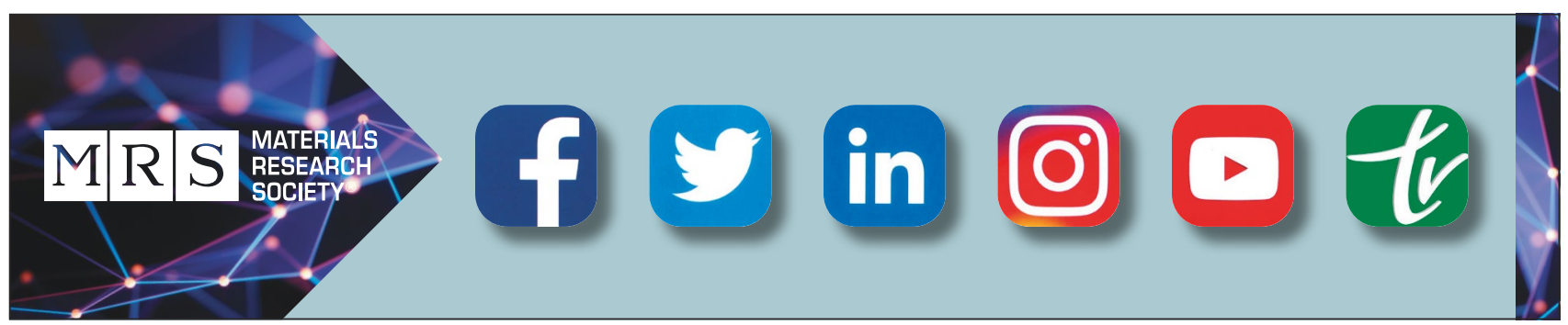

